“드 2014 IEEE. Personal use of this material is permitted. Permission from IEEE must be obtained for all other uses, in any current or future media, including reprinting/republishing this material for advertising or promotional purposes, creating new collective works, for resale or redistribution to servers or lists, or reuse of any copyrighted component of this work in other works." 


\title{
An Ultrasonic/RF GP-based Sensor Model Robotic Solution for Indoors/Outdoors Person Tracking
}

\author{
Daobilige $\mathrm{Su}$ \\ Centre for Autonomous System \\ University of Technology, Sydney \\ Email: daobilige.su@student.uts.edu.au
}

\author{
Jaime Valls Miro \\ Centre for Autonomous System \\ University of Technology, Sydney \\ Email: jaime.vallsmiro@uts.edu.au
}

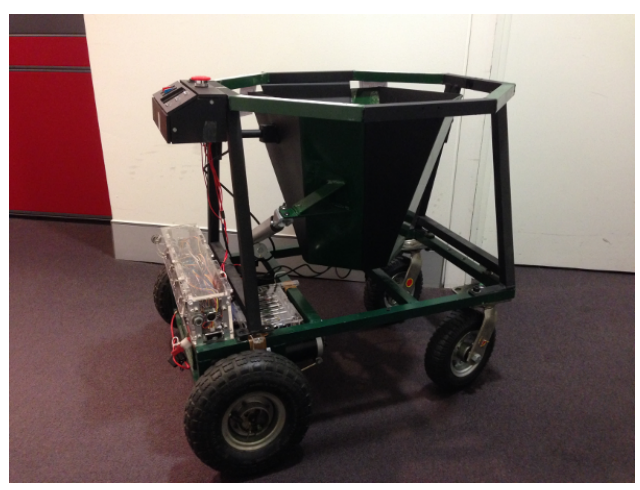

\begin{abstract}
An non-linear Bayesian regression engine for robotic tracking based on an ultrasonic/RF sensor unit is presented in this paper. The proposed system is able to maintain systematic tracking of a leading human in indoor/outdoor settings with minimalistic instrumentation. Compared to popular camera based localization system the sonar array/RF based system has the advantage of being insensitive to background light intensity changes, a primary concern in outdoor environments. In contrast to single-plane laser range finder based tracking the proposed scheme is able to better adapt to small terrain variations, while at the same time being a significantly more affordable proposition for tracking with a robotic unit. A key novelty in this work is the utilisation of Gaussian Process Regression (GPR) to build a model for the sensor unit, which is shown to compare favourably against traditional linear triangulation approaches. The covariance function yield by the GPR sensor model also provides the additional benefit of outlier rejection. We present experimental results of indoors and outdoors tracking by mounting the sensor unit on a Garden Utility Transportation System (GUTS) robot and compare the proposed approach with linear triangulation which clearly show the inference engine capability to generalise relative localisation of human and a marked improvement in tracking accuracy and robustness.
\end{abstract}

\section{Motivation}

The use of solutions to track mobile targets has been an active area of research for the robotics community in a wide range of application domains. Different successful applications have been developed for museum guidance [1], hospital assistance [2],pedestrian tracking [3] or outdoor human assistance [4]. Human localization and tracking systems are generally based on laser range finders, camera or combinations of these [5] [6] [7] [8] [9] [10]. These are well understood sensors that offer readily available measurements easy to integrate into standard tracking solutions. However, off-theshelf cameras tend to behave poorly when background lighting conditions change, while standard IR-based RGB-D cameras become inoperative under direct exposure to sun light, whereas laser based solutions, while significantly more accurate and resilient to outdoor lighting conditions, can be rather costly and potentially unwieldy mechatronic contraptions when design to operate in uneven terrains.

This work proposes an alternative ultrasonic-based sensor unit to localize a leading guide relative to a mobile robot for continuous tracking. This is particular driven by the need of an affordable yet robust relative localization system for an assistant robotic gardening unit operating on uneven outdoor
Fig. 1. Garden Utility Transportation System (GUTS), which is used to assist gardeners by following them while carrying a heavy dustbin

terrains. The Garden Utility Transportation System (GUTS), shown in Fig 1, is a differential drive mobile robot system fitted with an auto-tipping mechanism designed to aid a professional landscaper/gardener in transporting and manipulating garden waste in an outdoor setting, thus preventing them from repetitive manipulation of large loads in this risky occupational group. GUTS is designed to be able to follow a user in an outdoor environment, possibly rough and uneven, at a reasonable distance not to encroach on the user's personal space and working envelop, but still ensuring steady sensor readings.

Compared to traditional sonar array sensors which use Time Of Flight (TOF) and triangulation to find the relative location of a target with respect to the source, Gaussian Process Regression techniques are proposed in this work to improve the predicted accuracy of the reference target. This is predicated on the ability of these Bayesian engines to capture noisy non-linear relationships. Sonar range finders are notoriously noisy and difficult to synchronize precisely. Individual sensor performance tends to differ appreciably due the non-linearity of their readings, which are hard to capture by standard linear sensor models. As such accuracy of customary linear triangulation methods based on sonar TOF's measurements tend to be generally poor and will only degrade with time. GPRs on the other hand are trained with real data obtained by the actual individual sensor, thus training data has already taken into consideration issues such as poorly synchronized signal transmission, system delays or subtle difference in individual 


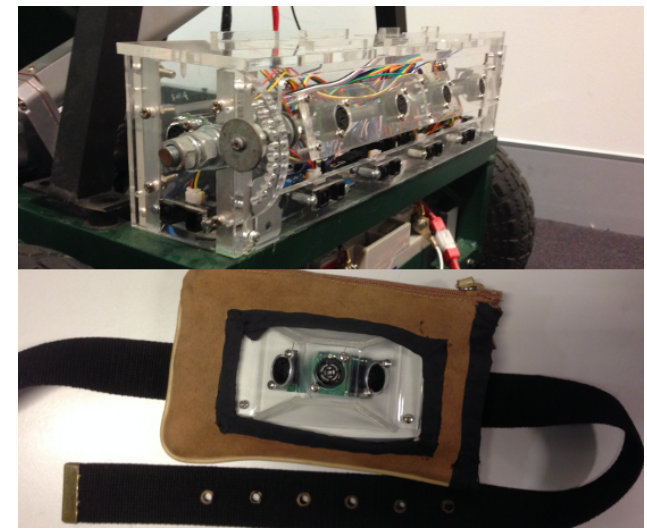

Fig. 2. Sonar array on GUTS for ultrasonic signal measurements (above) and human-carrying POD with transmitting ultrasonic arrangement (below)

sensor behaviours.

The structure of this paper is as follows: in Section II, related work about human localization and robotic tracking systems is presented. Section III describes the proposed robotic sensor unit and associated software framework while Section IV outlines the proposed sensor model methodology. Results from a set of indoor and outdoor experiments will be shown in Section V to demonstrate the performance of GPRs in particular when compared with a canonical triangulation technique. Section VI will present the concluding remarks.

\section{RELATED WORK}

There are a variety of robot relative human localization systems reported in the literature. They often differ in the selection of sensors and features that they extract to accomplish the tracking task, as well as the actual smoothing technique. Aggarwal et al. [11] provide an overview of interpreting and tracking human motions using cameras. The methods reported extract and track features such as color, texture, or shape contours. Subsequently, various filtering techniques are used to estimate the target's state. Gockley et al. [8] make use of a laser based approach to implement natural personfollowing behaviours in their social robots. Miro et al. [10] compared laser and camera information in estimating indoor the human relative localization for tracking. It was clearly demonstrated how camera based approaches could be easily influenced by variations in background lighting, even under steady indoor conditions, whereas laser range finders offered good performance at relatively high cost.

Beyond the sphere of customary laser/camera based approaches, other sensors have also been employed in tracking system. Dang et al [12] utilised an infrared camera to localize and track the human. Hong et al. [13] made use of structured lighting range sensors to detecting and tracking people with a mobile robot. Chang et al. [14] introduced ultra-wideband (WUB) radar for human detection and tracking. Nakamura et al. [15] use a microphone array for sound source localization and human tracking. This approach exploited the speed of sound and differences in the time of arrival of the human sound

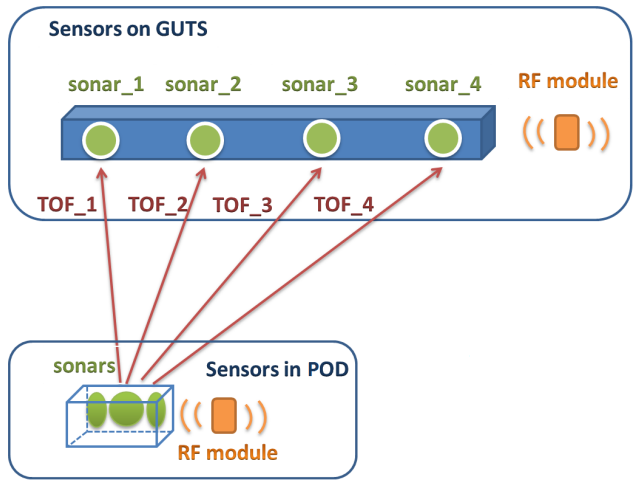

Fig. 3. Sensor configurations on GUTS and human carrying POD

signals to identify the sound source, but it required a dedicated multichannel synchronized sampling board. Moreover, it requires that people keep talking to be located accurately. Hollinger et al. [16] introduced ranging radio for tracking a moving target.

Wilhelm et al. [17] proposed a sensor fusion technique with vision and sonar for people tracking with a mobile robot. In their case sonar was uses to add range information to the visual content. Kohler et al. [18] also used ultrasonic sensors and joined particle weighting for vehicle tracking. Ullah et al. [19] employed a sonar array for tracking a target. In their approach, the sonar array fires and receives along multiple paths, yet ultimately their solution can potentially loose performance accuracy when the target sits outside the path of the sonar beam directions.

\section{ULTRASOUND/RF SENSOR UNIT}

The proposed ultrasonic/RF sensor unit modules for the robotic tracking system are depicted in Fig 2 as mounted on GUTS. Fig. 3 represents a block diagram of its operating functionality. It consists of two parts:

- An active sonar emitter array in a human carrying Portable User Device (POD). This part consists of three sonar sensors actively emitting ultrasonic pressure waves. No TOF measurement are computed by these sensors. At the time of firing, the RF module on the POD also emits a faster RF signal.

- A passive sensor array attached to the GUTS unit. It consists of four sonar units equally spaced in front of the GUTS robot. The sonar sensors operate in passive mode listening for signal from other sources. An RF module is used to determine when to start listening for incoming signals. Once the RF module on the GUTS sonar array receives the RF signal from the POD indicating the beginning of ultrasound transmission, it start a timer. The time lapsed from the timer start until the each sonar unit measures an incoming signal is the corresponding TOF for that sonar.

\section{A. Sensor Units}

The Sharp SRF02 ultrasonic sensor employed in this work is shown in Fig. 4, alongside its effective beam characteristics. 

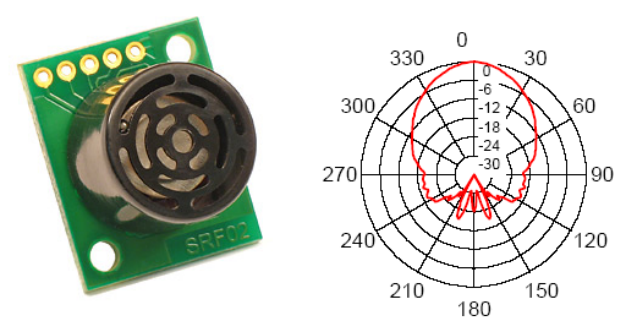

Fig. 4. SRF02 sonar sensor and its beam characteristics.

The SRF02 exhibits a pattern that covers around 60 degrees in the horizontal plane. In order to cover a wider area to allow improved freedom of mobility to the user we employ 3 timesynchronised SRF02 sensor units in the human carrying POD in the arrangement shown at the bottom of Fig 3. In such setup, the sonar sensors in the POD can cover approximately 180 degrees allowing more robust user tracking. An Arduino in GUTS is connected to the four sonar sensors via the I2C communication bus and sends TOF data to a control unit via serial communication.

A pair of $433 \mathrm{MHz} \mathrm{RF}$ transmitter/receiver units are used to time-synchronise firing the sonar sensor array both in GUTS and the POD, each coordinated by an Arduino microcontroller. As explained above, there is an alternate sequence of acoustic listening in GUTS and emitting of the acoustic signal by the POD unit. An RF module is needed to coordinate the emitting and receiving protocol of these sonar measurements as relying on synchronization of system time in both microcontroller is not accurate enough; the timer/crystal performance on both microcontroller could not possibly be identical. Under normal circumstances that would not be a major issue, however given the speed of sound a small difference in the two timers (generally in terms of a few micro seconds per second) can steadily accumulate to bigger value and have a significant big impact on TOF measurement when typical TOF readings are in the order of a few thousand micro seconds. Under these conditions localization drifting is clearly apparent. Compared to the propagation of acoustic waves in air, the transmission of RF signals are relatively fast and can be ignored. While there will be some physical delay in the RF transmission, it can be regarded as constant and taken into account by the training model described below.

\section{B. Software Setup}

The Robot Operating System (ROS) middleware has been employed as the software framework for the GUTS platform. A ROS package was developed with nodes to collect sonar ToF sensor measurements, computing the GPR prediction, calculate control commands and sending motor driver messages to the low-level motor controllers. Standard GUI packages in ROS such as Rviz provide feedback about the relative location of the POD-carrying human inferred by GPR at any one time. Learning the GPR sensor model and on-line regression was accomplished with the Gaussian Process Machine Learning (GPML) toolbox in MATLAB, linked to ROS via the
MATLAB-ROS bridge package.

\section{GPR SENSOR MODUle}

Given the TOF measurement with the sonar array on GUTS, the slight delay introduce by the RF transmission and the different non-linear response performance of each sonar sensor, standard performance of triangulation method suffers. In this work we propose a learning mechanism based on non-linear Bayesian Gaussian Processes to encapsulate the influence of these factor into a non-linear regression model. A Gaussian Process is a collection of random variables, any finite number of which have (consistent) joint Gaussian distributions [20]. The GPR is described with following equations:

$$
\begin{gathered}
\mathbf{f} \sim \mathcal{N}(\boldsymbol{\mu}, \boldsymbol{\Sigma}) \\
{\left[\begin{array}{c}
\mathbf{f} \\
\mathbf{f}_{*}
\end{array}\right] \sim \mathcal{N}\left(\left[\begin{array}{c}
\boldsymbol{\mu} \\
\boldsymbol{\mu}_{*}
\end{array}\right],\left[\begin{array}{cc}
\boldsymbol{\Sigma} & \boldsymbol{\Sigma}_{*} \\
\boldsymbol{\Sigma}_{*}^{\mathbf{T}} & \boldsymbol{\Sigma}_{* *}
\end{array}\right]\right)} \\
\mathbf{f}_{*} \mid \mathbf{f} \sim \mathcal{N}\left(\boldsymbol{\mu}+\boldsymbol{\Sigma}_{*}^{T} \boldsymbol{\Sigma}^{-1}(\mathbf{f}-\boldsymbol{\mu}), \boldsymbol{\Sigma}_{* *}-\boldsymbol{\Sigma}_{*}^{T} \boldsymbol{\Sigma}^{-1} \boldsymbol{\Sigma}_{*}\right)
\end{gathered}
$$

Eq. 1 describes the general form of GPR, where the function output $\mathbf{f}$ is a joint distribution over data mean $\boldsymbol{\mu}$ and covariance $\Sigma$.

$\boldsymbol{\mu}, \boldsymbol{\Sigma}$ and $\mathbf{f}$ represent mean, covariance and output of training data. $\boldsymbol{\mu}_{*}, \boldsymbol{\Sigma}_{*} *$ and $\mathbf{f}_{*}$ represent mean, covariance of test data and the output prediction. $\boldsymbol{\Sigma}_{*}^{T}$ represent the spatial covariance between training and test data. Eq. 3 describes the predicted mean $\boldsymbol{\mu}+\boldsymbol{\Sigma}_{*}^{T} \boldsymbol{\Sigma}^{-1}(\mathbf{f}-\boldsymbol{\mu})$ and covariance $\boldsymbol{\Sigma}_{* *}-\boldsymbol{\Sigma}_{*}^{T} \boldsymbol{\Sigma}^{-1} \boldsymbol{\Sigma}_{*}$.

A zero mean function

$$
\mu_{i}=m\left(x_{i}\right)=0
$$

and squared exponential function with noisy observation

$$
\Sigma_{i j}=k\left(x_{i}, x_{j}\right)=\sigma_{f}^{2} \exp \left(-\frac{1}{2 \ell^{2}}\left(x_{i}-x_{j}\right)^{2}\right)+\sigma_{n}^{2} \delta_{i i^{\prime}}
$$

have been used in our GPR model. We used exact inference method to do the optimization and obtained the set of hyperparameters $\sigma_{f}, \ell$ and $\sigma_{n}$ fully describing the GPR. $\delta_{i i^{\prime}}$ is 1 when $i=i^{\prime}$ and 0 otherwise.

We use the GPR methodology to build a sensor model for each sonar unit. Four independent GPRs are trained and a sensor models thus derived for each of the four sonars describing the likelihood of attaining a given sonar measurement at a certain location. The set up for obtaining training data is shown in Fig. 5. We captured $20 \mathrm{ToF}$ data points at each (orange) grid point to training each GPR. The laser range finder was used to derive the ground truth of the POD (x,y) location.

After training, we end up with the following GPR sensor models

$$
\begin{aligned}
& P\left(S_{1} \mid x, y\right)=G P R_{1}\left(S_{1} \mid x, y\right) \\
& P\left(S_{2} \mid x, y\right)=G P R_{2}\left(S_{2} \mid x, y\right) \\
& P\left(S_{3} \mid x, y\right)=G P R_{3}\left(S_{3} \mid x, y\right) \\
& P\left(S_{4} \mid x, y\right)=G P R_{4}\left(S_{4} \mid x, y\right)
\end{aligned}
$$




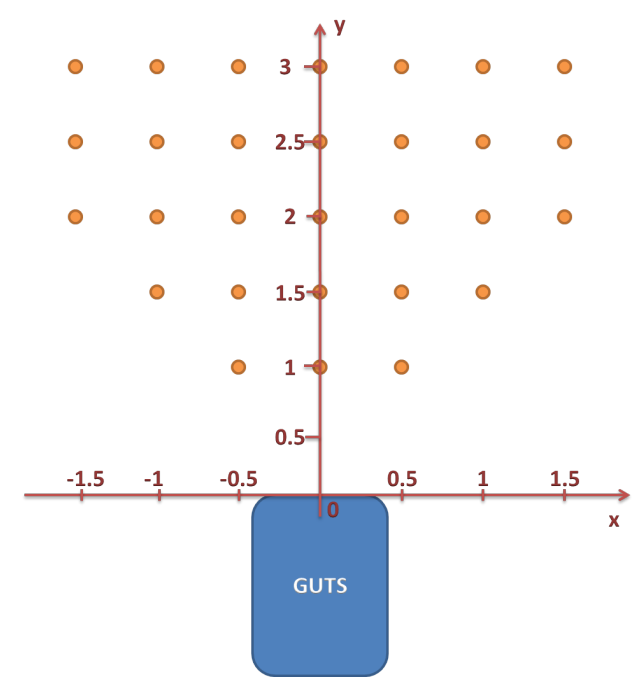

Fig. 5. GPR training setup: 20 sonar array measurements, each of them consisting of 4 TOF data taken at each orange point. Exact (x,y) positions extracted from laser range finder data are used as ground truth in the training of the GPR based sensor model for each sonar.

where $S_{1}$ to $S_{4}$ are the expected sensor readings at a certain $(\mathrm{x}, \mathrm{y})$ position.

Given an $(\mathrm{x}, \mathrm{y})$ location of interest, the GPR provides four expected sensor readings with corresponding covariances, making this type of sensor model a suitable proposition to be employed within the framework of a typical tracking filter such as particle filters, where the prior probability of each particle can be effectively weighted by the sensor model to calculate a posterior probability, as:

$$
\begin{aligned}
& P_{\text {post }}\left(x, y \mid S_{1}, S_{2}, S_{3}, S_{4}\right)= \\
& \frac{P\left(S_{1}, S_{2}, S_{3}, S_{4} \mid x, y\right) P_{\text {prior }}(x, y)}{P\left(S_{1}, S_{2}, S_{3}, S_{4}\right)}= \\
& \frac{\left(P\left(S_{1} \mid x, y\right) P\left(S_{1} \mid x, y\right) P\left(S_{1} \mid x, y\right) P\left(S_{1} \mid x, y\right)\right) P_{\text {prior }}(x, y)}{P\left(S_{1}, S_{2}, S_{3}, S_{4}\right)}
\end{aligned}
$$

where $P\left(S_{1} \mid x, y\right)$ to $P\left(S_{4} \mid x, y\right)$ would be characterised by the sensor models derived from the training (Eq. 6). Since $S_{1}$ to $S_{4}$ are our actual observation, $P\left(S_{1}, S_{2}, S_{3}, S_{4}\right)=1$.

After the training phase, a regression function for each of the four sonar sensor modules will be obtained which estimates the expected sensor reading and associated spatial covariance at a certain $(\mathrm{x}, \mathrm{y})$ location. The mean and covariance function for one of the sonars is shown in Fig. 6 and Fig. 7. It can be observed from the figure that the area where we have a good spread of training points exhibits a covariance in the region of 2000 micro seconds or less. This correspond to a standard deviation around 40 micro seconds, which is within the operational characteristics of the type of sonar sensors used in this work. The mean and covariance function for other three sensors are similar and have been omitted.

In the work presented here for inference an unknown prior probability $P_{\text {prior }}(x, y)$ is assumed, represented as a uniform distribution, hence Eq. 7 can be further simplified as

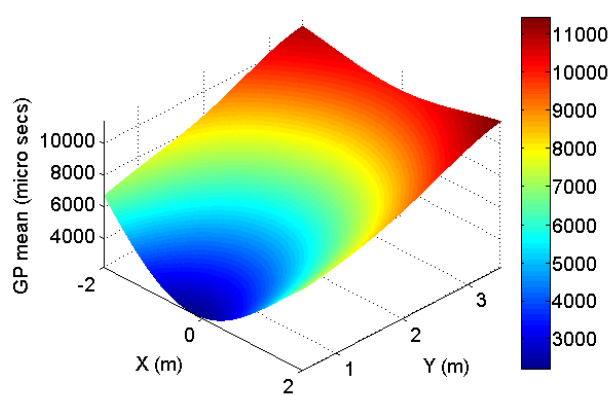

Fig. 6. Mean function of GPR for sonar 1

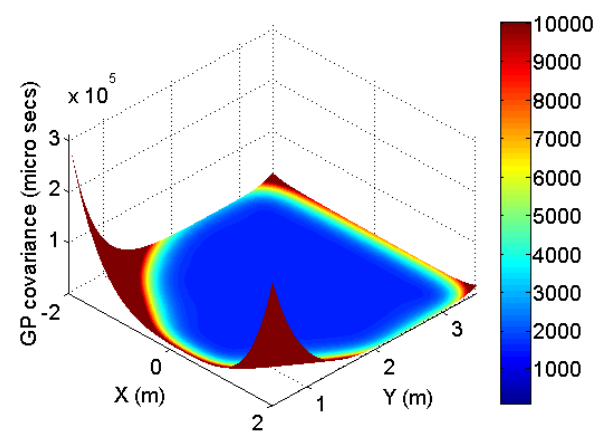

Fig. 7. Covariance function of GPR for sonar 1

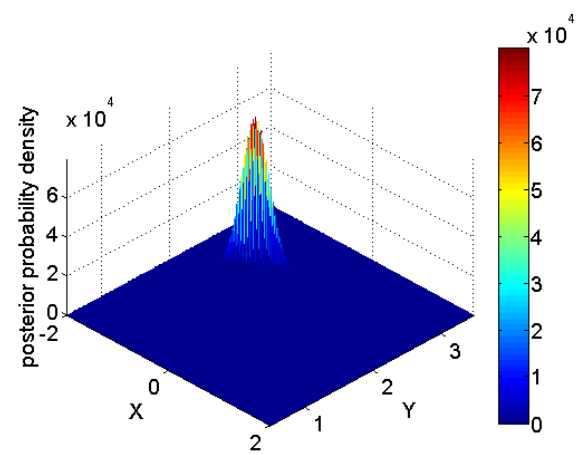

Fig. 8. Example of the probability density map of a TOF observation data. The $(\mathrm{x}, \mathrm{y})$ position with the highest probability is selected as the predicted location.

$$
\begin{aligned}
& P_{\text {post }}\left(x, y \mid S_{1}, S_{2}, S_{3}, S_{4}\right)= \\
& P\left(S_{1} \mid x, y\right) P\left(S_{1} \mid x, y\right) P\left(S_{1} \mid x, y\right) P\left(S_{1} \mid x, y\right)
\end{aligned}
$$

For each observation $S_{1}$ to $S_{4}$, a probability density map such as the one shown in Fig. 8 can be derived using Eq. 8 . The $(\mathrm{x}, \mathrm{y})$ position with highest probability can then be chosen as the predicted location for the measured ultrasound combination. Furthermore, given the measure of uncertainty associated to our predictions, if an expected sensor readings for a given ( $\mathrm{x}, \mathrm{y}$ ) falls outside the $1 \mathrm{x} \sigma$ region at the location with the overall highest probability, the prediction is rejected as a likely outlier possibly caused by a false sensor reading or bounced ultrasonics signal. 


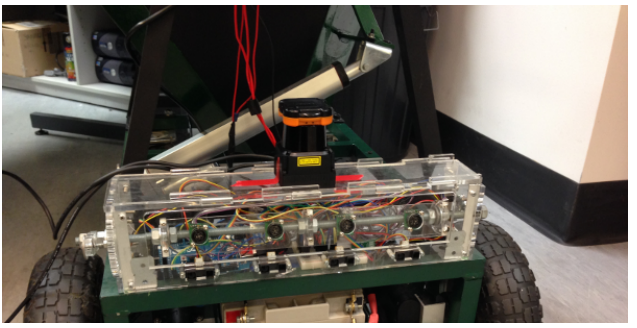

Fig. 9. Experimental setup for system validation with the laser range finder mounted at the front of the vehicle.

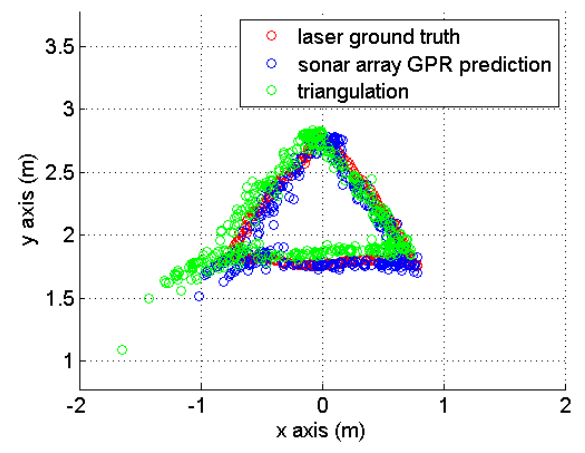

Fig. 10. Experimental result of POD following triangular trajectory.

\section{EXPERIMENTAL RESUlTS}

In order to validate the accuracy of the sonar/RF robotic tracking system, an experiment was first devised whereby given a stationary GUTS platform, a person carrying the POD would walk around the robot following certain trajectory patterns. Laser scans measurements where taken simultaneously with a Hokuyo laser range finder added to the front of GUTS as shown in Fig. 9. The true location of the user with respect to the robot was then derived from the analysis of the laser range data scans.

Results from the GPR inference are shown in Fig. 10 for a triangular shape trajectory example. Blue circles represent GPR regression while red circles are the corresponding ground truth data gathered from the laser range finder. Green circles denote predictions based on simple triangulation. From the figure it can be observed how the accuracy of the proposed GPR-based sensor model is markedly improved. Figs. 11 and 12 provide a qualitatively indication of the predictive errors given a triangulation methodology and the proposed GPR prediction approach in relation to the laser ground truth data. In both figures the " $y$ " axis represents the user locations based on the laser data, and the " $x$ " axis corresponds to the values calculated by triangulation and GPR. It can be observed how GPR predictions, while not perfect, are able to stay closer to the ideal 45 degree slope, marked as a red lines in both figures, while triangulation exhibits a clear shift and wider dispersion.

The above tests were repeated a number of times following arbitrary trajectories to quantitatively evaluate the accuracy of the predictions. Fig. 13 and 14 show the inaccuracies of the predictions along the " $\mathrm{x}$ " and " $y$ " axes for two examples

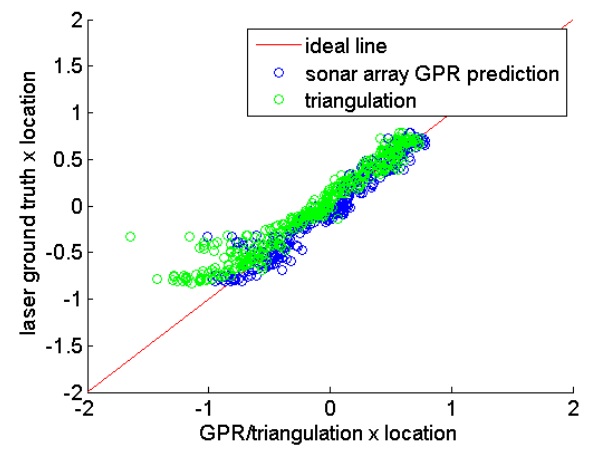

Fig. 11. Predicted " $\mathrm{x}$ " position by GPR/triangulation with respect to laser ground truth.

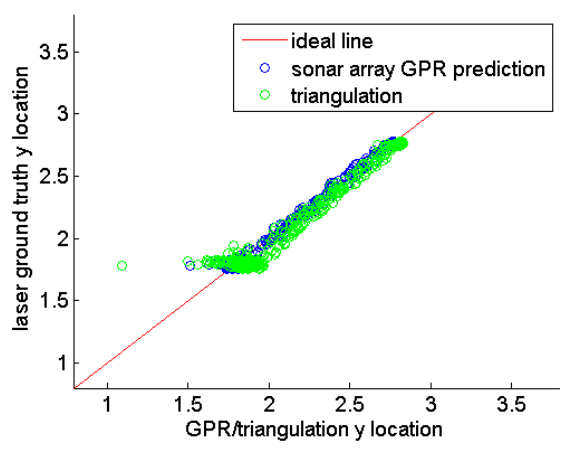

Fig. 12. Predicted "y" position by GPR/triangulation with respect to laser ground truth.

(the former corresponds to the triangular trajectory depicted in Fig 10) by depicting the mean predictive error of the POD with for the proposed GPR approach and the standard triangulation technique. Given the noisy measurements is not possible to provide a general absolute error figure, yet a clear trend of improved accuracy can be observed for the case of the GPR model when compared with the standard triangulation methodology in both $\mathrm{x}$ and $\mathrm{y}$ direction. To a large extent this is due to the ability of the GPR machinery to accommodate for the TOF noise in the acoustic data and the sensor nonlinearities.

In order to further demonstrate the ability of the proposed methodology to be integrated within a robotic platform as a viable and robust solution to track a human beacon, field tests of the GUTS platform following a person in a large indoor environment and in an outdoor setting were also carried out. The field tests were recorded and have been submitted as an attachment to this paper. Two accounts of the field trial's proceedings are illustrated in Figs. 15 and 16. In the field tests a simple linear proportional controller in translation and orientation velocity was implemented, yet that was sufficient to concluded that under normal walking pace and patterns GUTS was able to effectively follow the leading person, as shown in the attached videos. 


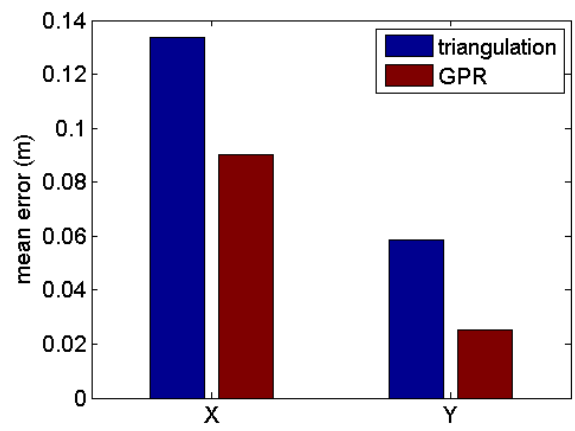

Fig. 13. Mean error in "x" and " $y$ " using triangulation and the GPR based approach for the triangular trajectory depicted in Fig. 10

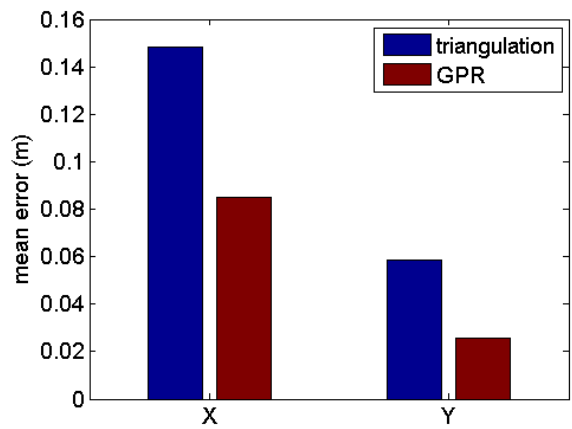

Fig. 14. Mean error in "x" and " $y$ " using triangulation and the GPR based approach for a different trajectory experiment.

\section{CONCLUSIONS}

An ultrasonic/RF sensor unit able to maintain systematic tracking of a leading guide with the help of a non-linear Bayesian regression engine has been presented in this paper. The proposed GPR approach has been proven to have the ability to handle the noise and non-linearities associated to measurements from such sensor package, inherently more significant than those expected from comparable solutions from sensors such as cameras or laser range finders. In doing so, the proposed approach has the fundamental advantage of being insensitive to background lighting conditions, a primary concern in outdoor settings, and also better suited to deal with terrain variations when compared to more costly single laser based solutions. The accuracy of the proposed technique has also been proven to compare favourably with a standard triangulation methodology.

\section{ACKNOWLEDGEMENT}

The authors would like to thank Mr. Luke Di Palma, Mr. Samuel Klistorner and Mr. Snehal Ashok Tangadpalliwar for their preliminary researches.

\section{REFERENCES}

[1] S. Thrun, M. Bennewitz, W. Burgard, A. Cremers, F. Dellaert, D. Fox, D. Haehnel, C. Rosenberg, N. Roy, J. Schulte and D. Schulz, "Minerva: A second generation mobile tour-guide robot", in Proc. of the IEEE International Conference on Robotics and Automation (ICRA 1999), 1999.

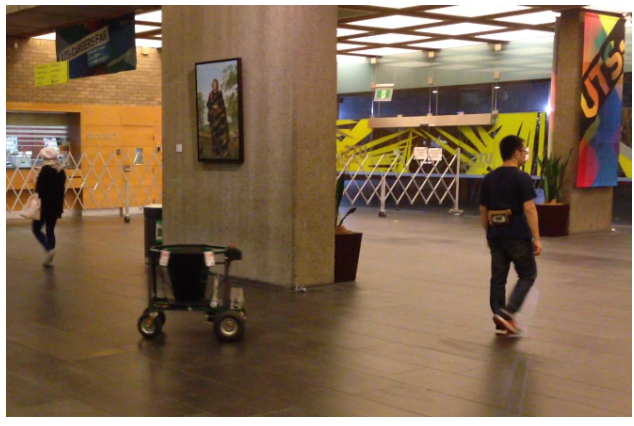

Fig. 15. Snapshot of the robotic tracking field test of the proposed ultrasonic/RF sensor unit and GPR-based sensor modelling approach in an indoor environment.

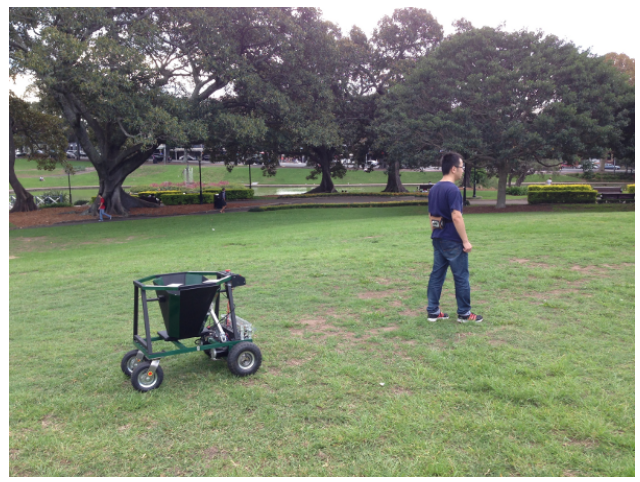

Fig. 16. Snapshot of the robotic tracking field test with the proposed ultrasonic/RF sensor unit and GPR-based sensor modelling approach in outdoor terrain.

[2] J. F. Engelberger, "Health-care robotics goes commercial: The helpmate experience", in Robotica, vol. 11, 1993, pp. 517523.

[3] L. Davis, V. Philomin and R. Duraiswami, "Tracking humans from a moving platform", in Proc. of the International Conference on Pattern Recognition (ICPRO0), 2000.

[4] M. Kobilarov, G. Sukhatme, J. Hyams and P. Batavia, "People tracking and following with mobile robot using an omnidirectional camera and a laser", in Proc. of the IEEE International Conference on Robotics and Automation (ICRA 2006), 2006.

[5] Z. Chen and S. T. Birchfield, "Person following with a mobile robot using binocular feature-based tracking", IEEE/RSJ International Conference on Intelligent Robots and Systems (IROS 2007), 2007.

[6] T. Yoshimi, M. Nishiyama, T. Sonoura, H. Nakamoto, S. Tokura, H. Sato, F. Ozaki, N. Matsuhira and H. Mizoguchi, "Development of a Person Following Robot with Vision Based Target Detection", IEEE/RSJ International Conference on Intelligent Robots and Systems (IROS 2006) , 2006.

[7] H. O. Latif, N. Sherkat and A. Lotfi, "Fusion of automation and teleoperation for person-following with mobile robots", International Conference on Information and Automation (ICIA 2009), 2009.

[8] R. Gockley, J. Forlizzi and R. Simmons, "Natural person-following behavior for social robots", 2nd ACM/IEEE International Conference on Human-Robot Interaction (HRI 2007), 2007.

[9] J. Lee and P. Stone, "Person tracking on a mobile robot with heterogeneous inter-characteristic feedback", IEEE International Conference on Robotics and Automation, 2008 (ICRA 2008), 2008.

[10] J. V. Miro, J. Poon and S. Huang, "Low-cost visual tracking with an intelligent wheelchair for innovative assistive care", 12th International Conference on Control Automation Robotics \& Vision (ICARCV 2012), 2012.

[11] J. K. Aggarwal and Q. Cai, "Human motion analysis: a review", Computer Vision and Image Understanding, vol. 73, no. 3, pp. 428440, 1999.

[12] Q. K. Dang and Y. Suh, "Human-following robot using infrared camera", 
11th International Conference on Control, Automation and Systems (ICCAS 2011), 2011.

[13] Y. Hong, M. Jung, H. Myung, H. Lee, Y. Lee and S. Kim, "Detecting and tracking people by mobile robot using structured light range sensor", IEEE/RSJ International Conference on Intelligent Robots and Systems (IROS 2005), 2005.

[14] S. Chang, M. Wolf and J. W. Burdick, "Human detection and tracking via Ultra-Wideband (UWB) radar", IEEE International Conference on Robotics and Automation (ICRA 2010), 2010.

[15] K. Nakamura, K. Nakadai, F. Asano and G. Ince, "Intelligent Sound Source Localization and its application to multimodal human tracking", IEEE/RSJ International Conference on Intelligent Robots and Systems (IROS 2011), 2011.

[16] G. Hollinger, J. Djugash and S. Singh, "Tracking a moving target in cluttered environments with ranging radios", IEEE International Conference on Robotics and Automation (ICRA 2008), 2008.

[17] T. Wilhelm, H. J. Bhme and H. M. Gross, "Sensor fusion for vision and sonar based people tracking on a mobile service robot", in Proc. of the International Workshop on Dynamic Perception, 2002.

[18] P. Kohler, C. Connette and A. Verl, "Vehicle tracking using ultrasonic sensors \& joined particle weighting", IEEE International Conference on Robotics and Automation (ICRA 2013), 2013.

[19] I. Ullah, Q. Ullah, F. Ullah and S. Shin, "Integrated collision avoidance and tracking system for mobile robot", in Proc. of the International Conference on Robotics and Artificial Intelligence, 2012.

[20] C. E. Rasmussen and C. K. I. Williams, "Gaussian processes for machine learning", 2006. 